\title{
A NOTE ON STICKELBERGER ELEMENTS FOR CYCLIC P-EXTENSIONS OVER GLOBAL FUNCTION FIELDS OF CHARACTERISTIC P
}

\author{
KI-SEng TAN
}

\begin{abstract}
We prove a special case of Tate's refinement of a conjecture of Gross concerning the Stickelberger element associated to a cyclic extension over a global function field of characteristic $p$.
\end{abstract}

\section{Introduction}

In this note we make some initial progress toward Tate's refinement [8] of a conjecture of Gross [3] concerning the Stickelberger element associated to a cyclic extension over a global function field of characteristic $p$.

Recall that Gross's conjecture concerns the form of the Stickleberger element which is an element in the integral group ring of the Galois group for an abelian extension of a global field. In this note, we only consider the function field case. To be precise, let us fix a global function field $k$ of characteristic $p$, and let $S=\left\{v_{0}, \ldots, v_{n}\right\}$ and $T$ be fixed non-empty finite sets of places in $k$ such that $S$ and $T$ are disjoint.

Let $L / k$ be an abelian extension unramified outside $S$ and let $G=\operatorname{Gal}(L / k)$. For $v \notin S$, let $F r_{v}$ denote the Frobenius element in $G$. As modified by Gross, the associated Stickelberger element $\theta_{G}[3,7]$ is the unique element in the group ring $\mathbb{Z}[G]$ such that for every non-trivial ring homomorphism $\chi: \mathbb{Z}[G] \longrightarrow \mathbb{C}$,

$$
\chi\left(\theta_{G}\right)=L(\chi, 0),
$$

where, $L(\chi, s), s \in \mathbb{C}$ is the modified $L$-function defined by

$$
L(\chi, s)=\prod_{v \notin S}\left(1-\chi\left(F r_{v}\right) N(v)^{-s}\right)^{-1} \times \prod_{v \in T}\left(1-\chi\left(F r_{v}\right) N(v)^{1-s}\right), \quad(\operatorname{Re}(s)>1) .
$$

Received July 3, 2003.

2000 Mathematics Subject Classification : $11 S 40$.

The author was supported in part by the National Science Council of Taiwan (R.O.C.), NSC91-2115-M-002-001.

Key words and phrases. Stickelberger element, special values of abelian L-functions, Conjecture of Gross, Class numbers. 
In [3] Gross conjectures that if $I$ is the augmentation ideal of $\mathbb{Z}[G]$, then $\theta_{G}$ is in $I^{n}$ and is congruent to $h \cdot \mathcal{R}_{G}$ modulo $I^{n+1}$. Here $h=h_{S, T}$ is the $T$ modified class number of the $S$-integers of $k$ and $\mathcal{R}_{G}$ is the refined regulator. This conjecture is the analogue of the class number formula (interpreted as a statement about the order of vanishing of the zeta function at " $s=0$ " and the first non-zero term in its Taylor expansion).

In [3], the refined regulator is defined as an element in $I^{n} / I^{n+1}$. We instead choose to adopt Tate's definition [8] and define the refined regulator as an element in the group ring.

Here we describe Tate's definition of the refined regulator. First, for each $i$, let

$$
\operatorname{deg}_{i}: k_{v_{i}}^{*} \longrightarrow \mathbb{Z}
$$

be the local valuation map which sends the local parameters to 1 . Recall that $U_{S, T}$, the group of $S$-units which are congruent to 1 modulo $T$, is torsion free. Let $\left\{u_{j}\right\}_{1 \leq j \leq n}$ be a $\mathbb{Z}$-basis of it. Then the classical regulator equals

$$
\pm \mathrm{M}_{S} \cdot \operatorname{det}_{1 \leq i, j \leq n}\left(\operatorname{deg}_{i}\left(u_{j}\right)\right)
$$

where the sign is determined by the ordering of the basis and $\mathrm{M}_{S}$ is the index of $U_{S, T}$ as a subgroup of the free part of the $S$-units. We choose the ordering to have the positive sign. For $0 \leq i \leq n$, let $G_{i}$ be the decomposition subgroup of $v_{i} \in S$, and let

$$
f_{i}: k_{v_{i}}^{*} \longrightarrow G_{i}
$$

be the local reciprocity law homomorphism. These local homomorphisms are viewed as analogue of the local valuation maps, and the refined regulator is defined.

Definition 1.1. We define

$$
\mathcal{R}_{G}=\operatorname{det}_{1 \leq i, j \leq n}\left(f_{i}\left(u_{j}\right)-1\right) \in \mathbb{Z}[G] .
$$

The definition of $\mathcal{R}_{G}$ depends on the choice of $v_{0}$ in $S$.

For a subgroup $H$ of $G$, let $I_{H}$ be the kernel of the homomorphism $\mathbb{Z}[G] \longrightarrow$ $\mathbb{Z}[G / H]$ induced from the natural quotient homomorphism. The following lemma is obvious.

Lemma 1.1. ([8]) We have

$$
\mathcal{R}_{G} \in \prod_{1 \leq i \leq n} I_{G_{i}}
$$

From now on, we assume that $G$ is cyclic of order $p^{m}$ and

$$
m_{0} \leq m_{1} \leq \cdots \leq m_{n} \leq m,
$$

where

$$
p^{m_{i}}=\left[G: G_{i}\right], \quad i=0, \ldots n .
$$


Let

$$
N=p^{m_{0}}+\cdots+p^{m_{n-1}} .
$$

In [8], Tate, using the valuation criterion (see Lemma 2.1), proves that if $m_{n}=m-1$, then $\theta_{G}$ and $h \cdot \mathcal{R}_{G}$ are both in $I^{N}$ where $N$ is defined in (5), and conjectures that, in the case where $m_{0}=0$, they are congruent modulo $I^{N+1}$. Since $N \geq n$, this is a refinement of the conjecture of Gross. Note that in the case where $m_{0}>0$ the congruence might not hold ([6]) and a more subtle formulation is needed. For more discussions on Tate's refinement, see $[6,2,1]$.

Our main result (proved in Section 2) is that this congruence indeed holds for the " $m_{0}=0 "$ " case.

Theorem 1.1. If $m_{n}=m-1$ and $m_{0}=0$, then $\theta_{G}, h \cdot \mathcal{R}_{G} \in I^{N}$, and

$$
\theta_{G} \equiv h \cdot \mathcal{R}_{G} \quad\left(\bmod I^{N+1}\right) .
$$

Theorem 1.1 is proved through making direct use of the fact that $G$ is a $p$ group. This allows us to work on $\mathbb{Z}_{p}[G]$ which is just $\mathbb{Z}[G] \otimes_{\mathbb{Z}} \mathbb{Z}_{p}$. In particular, if we let

$$
I_{H, p}=I_{H} \otimes_{\mathbb{Z}} \mathbb{Z}_{p}
$$

then Theorem 1.1 is proved in Section 2 by using the valuation criterion (Lemma 2.1 ) in combination with the following result whose proof is postponed until Section 3 .

Theorem 1.2. If $G$ is a cyclic p-group, then

$$
\theta_{G} \in \prod_{1 \leq i \leq n} I_{G_{i}, p}
$$

and

$$
\theta_{G} \equiv h \cdot \mathcal{R}_{G} \quad\left(\bmod I_{G, p} \cdot \prod_{1 \leq i \leq n} I_{G_{i}, p}\right)
$$

\section{The valuation criterion}

We follow the notation in [8]. Assume that $G$ is cyclic of order $p^{m}$ and $G_{n}$ is of order $p^{m-1}$. Let $\sigma$ generate $G$ and put $\rho=\sigma^{p^{m-1}}$. Let $\chi$ be a character of $G$ of order $p^{m}$ so that $\zeta:=\chi(\sigma)$ is a primitive $p^{m}$ th root of unity. Put $\lambda=\zeta-1$. The proof of the following lemma can be found in [6].

Lemma 2.1. (Tate, $[8])$ For $j \geq 1$, the character $\chi$ induces an isomorphism

$$
\begin{aligned}
\chi: I^{j} \cap(\rho-1) \mathbb{Z}[G] / I^{j+1} \cap(\rho-1) \mathbb{Z}[G] & \stackrel{\sim}{\longrightarrow}(\lambda)^{j+p^{m-1}-1} /(\lambda)^{j+p^{m-1}} \\
(\sigma-1)^{j-1}(\rho-1) & \mapsto \lambda^{j+p^{m-1}-1}
\end{aligned}
$$

Assuming Theorem 1.2, we now easily prove Theorem 1.1. 
Proof. (of Theorem 1.1) From the assumption $m_{0}=0$ and $m_{n}=m-1$, we have

$$
\chi\left(\prod_{1 \leq i \leq n} I_{G_{i}, p}\right) \subseteq(\lambda)^{p^{m_{1}}+\cdots+p^{m_{n}}}=(\lambda)^{N+p^{m-1}-1},
$$

and

$$
\chi\left(I_{G, p} \cdot \prod_{1 \leq i \leq n} I_{G_{i}, p}\right) \subseteq(\lambda)^{1+p^{m_{1}}+\cdots+p^{m_{n}}}=(\lambda)^{N+p^{m-1}} .
$$

\section{The method of using $\mathbb{Z}_{p}$-extensions}

In this section, we will frequently use the following version of Local Leopoldt Theorem (cf. [5, 9]).

Lemma 3.1. Let $v$ be a place of $k$ and $\alpha \in k$. If $\alpha^{1 / p} \in k_{v}$, the $\alpha^{1 / p} \in k$.

Proof. If $\alpha^{1 / p} \notin k$, then $k\left(\alpha^{1 / p}\right)=k^{1 / p}$. But since $k$ is dense in $k_{v}$, we can not have $k^{1 / p} \subset k_{v}$.

Let $\Gamma_{S}$ be the Galois group of the maximal pro- $p$ abelian extension which is unramified outside $S$. In [5], it is proved that $\Gamma_{S}$ is a product of countably many copies of $\mathbb{Z}_{p}$ (see also [9]). Therefore, we can extend $L / k$ to an extension $E / k$ unramified outside $S$ such that $\operatorname{Gal}(E / k) \simeq \mathbb{Z}_{p}$.

For each $i=0, \ldots, n$, let $k_{i}$ and $E_{i}$ denote the completions at $v_{i}$ of $k$ and $E$ respectively. Put $\mathcal{N}_{i}=\operatorname{Norm}_{E_{i} / k_{i}}\left(E_{i}^{*}\right)$. Then $k_{i}^{*} / \mathcal{N}_{i}$ is either $\mathbb{Z}_{p}, \mathbb{Z}$, or $\{0\}$.

Let $\tilde{\Gamma}$ be the $p$-completion of the group $k^{*} \backslash \mathbb{A}_{k}^{*} / \prod_{i=1}^{n} \mathcal{N}_{i} \times \prod_{v \notin S} \mathcal{O}_{v}^{*}$. Here, as usual, $\mathbb{A}_{k}^{*}$ denotes the group of ideles and $\mathcal{O}_{v}^{*}$ denotes the group of local units at $v$. Then $\tilde{\Gamma}$ is the Galois group of the maximal abelian pro- $p$-extension of $k$ over which the decomposition group at each $v_{i}, i=1, \ldots, n$, is exactly the $p$-completion of $k_{i}^{*} / \mathcal{N}_{i}$. Note that here we impose no condition at $v_{0}$. We have the natural homomorphism $\Psi$ which maps the $p$-completion of $\prod_{i=1}^{n} k_{i}^{*} / \mathcal{N}_{i}$ to $\tilde{\Gamma}$.

Lemma 3.2. With the $\Psi$ as above, the following are true.

(1) The homomorphism $\Psi$ is injective and the $\mathbb{Z}_{p}$-module $\tilde{\Gamma} / \operatorname{Im}(\Psi)$ is torsion free.

(2) There exists an abelian pro-p-extension $F / k$ which satisfies the following.

(a) The field $F$ contains $E$.

(b) The Galois group $\Gamma:=\operatorname{Gal}(F / k)$ is isomorphic to $\mathbb{Z}_{p}^{d}$ for some $d$.

(c) The decomposition group $\Gamma_{i}$ at each $v_{i}$, for $i=1, \ldots, n$, is exactly the $p$-completion of $k_{i}^{*} / \mathcal{N}_{i}$.

(d) The natural homomorphism $\prod_{i=1}^{n} \Gamma_{i} \longrightarrow \Gamma$ is injective and the cokernel is torsion free.

Proof. We first prove that $\tilde{\Gamma}$ is $p$-torsion free. Then, by the fact that it is a quotient of $\Gamma_{S}$, it is also a product of countably many copies of $\mathbb{Z}_{p}$. Now, suppose that $\gamma \in \tilde{\Gamma}$ and $\gamma^{p}=1$. If $\gamma$ is represented by an idele $z=\left(z_{v}\right)$, then there is an $\alpha \in k^{*}$ and a $u \in \prod_{i=1}^{n} \mathcal{N}_{i} \times \prod_{v \notin S} \mathcal{O}_{v}^{*}$ such that $z^{p}=\alpha \cdot u$. In 
particular, in $k_{0}^{*}$ we have $z_{v_{0}}^{p}=\alpha$. By Lemma 3.1, we have $\alpha=\beta^{p}$ for some $\beta \in k^{*}$. Since $k_{0}^{*}$ and $k_{i}^{*} / \mathcal{N}_{i}, i=1, \ldots, n$ are $p$-torsion free, we have $z_{v_{i}} \beta^{-1}=1$, for $i=0, \ldots, n$. Also, we have $z_{v} \beta^{-1} \in \mathcal{O}_{v}^{*}$, for $v \notin S$. This implies that $\gamma=1$.

For $i=1, \ldots, n$, let $x_{i} \in k_{i}^{*}$, and let $\bar{x}$ be the element determined by $\left(x_{1}, \ldots, x_{n}\right)$ in the $p$-completion of $\prod_{i=1}^{n} k_{i}^{*} / \mathcal{N}_{i}$. If $\bar{x}$ is in the kernel of $\Psi$, then for each $j=1,2, \ldots$, there exists $\alpha_{j} \in K^{*}, u(j) \in \prod_{i=1}^{n} \mathcal{N}_{i} \times \prod_{v \notin S} \mathcal{O}_{v}^{*}$ and $y(j) \in \mathbb{A}_{k}^{*}$ such that in $\mathbb{A}_{k}^{*}$,

$$
\left(x_{1}, \ldots, x_{n}\right)=\alpha_{j} \cdot u(j) \cdot y(j)^{p^{j}} .
$$

In particular, we have

and by Lemma 3.1, we have

$$
\alpha_{j} \in\left(k_{0}^{*}\right)^{p^{j}}
$$

$$
\alpha_{j} \in\left(k^{*}\right)^{p^{j}}
$$

Consequently, the element $\bar{x}$ is $p$-divisible in the $p$-completion of $\prod_{i=1}^{n} k_{i}^{*} / \mathcal{N}_{i}$, hence is trivial. This shows that $\Psi$ is injective. If there is a $\gamma \in \tilde{\Gamma}$ obtained from an idele $z=\left(z_{v}\right)$ such that $\gamma^{p}=\Psi(\bar{x})$, then for each $j=1,2, \ldots$, there are $\alpha_{j} \in K^{*}, u(j) \in \prod_{i=1}^{n} \mathcal{N}_{i} \times \prod_{v \notin S} \mathcal{O}_{v}^{*}$ and $y(j) \in \mathbb{A}_{k}^{*}$ such that

$$
\left(x_{1}, \ldots, x_{n}\right)=z^{p} \cdot \alpha_{l} \cdot u(j) \cdot y(j)^{p^{j}}
$$

Again, by Lemma 3.1, we have

$$
\alpha_{j} \in\left(k^{*}\right)^{p}
$$

and consequently, there is a $\bar{w}$ in the $p$-completion of $\prod_{i=1}^{n} k_{i}^{*} / \mathcal{N}_{i}$ such that $\bar{x}=\bar{w}^{p}$. Therefore, $\gamma \cdot \Psi(\bar{w})^{-1}$ is in the $p$-torsion of $\tilde{\Gamma}$. Since $\tilde{\Gamma}$ is $p$-torsion free, we have $\gamma=\Psi(\bar{w})$. This completes the proof of (1). Also, since $\tilde{\Gamma}$ is a product of countably many copies of $\mathbb{Z}_{p}$, then by (1), we can find an abelian pro-p-extension $F_{0} / k$ which satisfies conditions (b), (c), (d). Then we put $F=F_{0} E$.

Now we pay off our last debt and prove Theorem 1.2.

Proof. (of Theorem 1.2) Let $F / k$ satisfy conditions (a), (b), (c), (d), in Lemma 3.2 , and let $\Gamma=\operatorname{Gal}(F / k)$. Since $\theta_{G}$ is functorial with respect to $G$, through the projective limit, we can define the Stickelberger element $\theta_{\Gamma} \in \mathbb{Z}[[\Gamma]]([9])$. Also, the refined regulator $\mathcal{R}_{\Gamma} \in \mathbb{Z}[[\Gamma]]$ is defined. If there is an $i \in\{1, \ldots, n\}$ such that $k_{i}^{*} / \mathcal{N}_{i}=\{0\}$, then $v_{i}$ splits completely and (see [3])

$$
\theta_{\Gamma}=0=\mathcal{R}_{\Gamma},
$$

and there is nothing to prove. We assume that $k_{i}^{*} / \mathcal{N}_{i} \neq\{0\}$, for every $i=$ $1, \ldots, n$. Then $\mathbb{Z}_{p} \Gamma_{i} \simeq \mathbb{Z}_{p}$. Choose a $\mathbb{Z}_{p}$-basis $\gamma_{1}, \ldots, \gamma_{d}$ of $\Gamma$, such that $\mathbb{Z}_{p} \Gamma_{i}=$ $\mathbb{Z}_{p} \gamma_{i}$, for $i=1, \ldots, n$, and put $t_{i}=\gamma_{i}-1$ for $i=1, \ldots, d$. Then in this case, the ring $\mathbb{Z}_{p}[[\Gamma]]$ is just the formal power series ring $\mathbb{Z}_{p}\left[\left[t_{1}, \ldots, t_{d}\right]\right]$ and the ideals $\bar{I}_{\Gamma, p}:=\operatorname{ker}\left(\mathbb{Z}_{p}[[\Gamma]] \longrightarrow \mathbb{Z}_{p}\right)$ and $\bar{I}_{\Gamma_{i}, p}:=\operatorname{ker}\left(\mathbb{Z}_{p}[[\Gamma]] \longrightarrow \mathbb{Z}_{p}\left[\left[\Gamma / \Gamma_{i}\right]\right]\right)$, $i=1, \ldots, d$, of $\mathbb{Z}_{p}[[\Gamma]]$ are just $\left(t_{1}, \ldots, t_{d}\right)$ and $\left(t_{i}\right)$. Here, as before, the natural ring homomorphisms are induced from the quotient homomorphisms

$$
\Gamma \longrightarrow\{0\},
$$


and

$$
\Gamma \longrightarrow \Gamma / \Gamma_{i}
$$

For each $i$, let $F_{i}$ be the fixed field of $\Gamma_{i}$. Since $v_{i}$ splits completely in $F_{i}$, the natural homomorphism $\mathbb{Z}_{p}[[\Gamma]] \longrightarrow \mathbb{Z}_{p}\left[\left[\Gamma / \Gamma_{i}\right]\right]$, maps both $\theta_{\Gamma}$ and $h \cdot \mathcal{R}_{\Gamma}$ to zero. This shows that as power series, both $\theta_{\Gamma}$ and $h \cdot \mathcal{R}_{\Gamma}$ are divisible by $t_{i}$ for every $i \in\{1, \ldots, n\}$. Therefore, we have

$$
\theta_{\Gamma} \in t_{1} t_{2} \ldots t_{n} \cdot \mathbb{Z}_{p}\left[\left[t_{1}, \ldots, t_{d}\right]\right]=\prod_{i=1}^{n} I_{\Gamma_{i}, p} .
$$

Applying the quotient map $\Gamma \longrightarrow G$, we get the inclusion (7).

In this case, Gross's Conjecture has been proved [9], so that $\theta_{\Gamma}-h \cdot \mathcal{R}_{\Gamma}$ is in $I_{\Gamma, p}^{n+1}$. Since it is also divisible by $t_{1} \ldots t_{n}$, we have

$$
\theta_{\Gamma}-h \cdot \mathcal{R}_{\Gamma} \in I_{\Gamma, p} \cdot \prod_{i=1}^{n} I_{\Gamma_{i}, p} .
$$

Congruence (8) then can be obtained by applying the quotient map $\Gamma \longrightarrow G$.

\section{References}

[1] Aoki, N. On Tate's refinement for a conjecture of Gross and its generalization. to appear in Journal de Theorie des Nombres Bordeaux, 2003.

[2] Burns, D. On relations between derivatives of abelian L-functions at $s=0$. Preprint, 2002

[3] Gross, B. On the values of abelian L-functions at $s=0$. J. Fac. Sci. Univ. Tokyo Sect. IA, Math. 35 (1988), 177-197.

[4] Hayes, D. The refined $p$-adic abelian Stark conjecture in function fields. Invent. Math. 94 (1988), 505-527.

[5] Kisilevsky, H. Multiplicative independence in function fields. J. Number Theory 44 (1993), $352-355$

[6] Lee, J. Stickelberger elements for cyclic extensions and the order of vanishing of abelian $L$-functions at $s=0$. to appear in Comp. Math.

[7] Tate, J. Les Conjectures de Stark sur les Fonctions L d'Artin en $s=0$, Birkhauser, 1984

[8] Tate, J. a letter to Joongul Lee, 1997

[9] Tan, K.-S. On the Special Values of Abelian L-Function. J. Math. Sci., Univ. Tokyo 1 (1995), 305-319.

Department of Mathematics, National Taiwan University, Taipei, 106, Taiwan

E-mail address: tan@math.ntu.edu.tw 